\title{
REVIEW
}

\section{Chronic subdural haematoma in the elderly}

\author{
V Adhiyaman, M Asghar, K N Ganeshram, B K Bhowmick
}

Postgrad Med J 2002;78:71-75

Chronic subdural haematoma is predominantly a disease of the elderly. It usually follows a minor trauma. A history of direct trauma to the head is absent in up to half the cases. The common manifestations are altered mental state and focal neurological deficit. Neurological state at the time of diagnosis is the most important prognostic factor. Morbidity and mortality is higher in the elderly but outcome is good in patients who undergo neurosurgical intervention.

See end of article for authors' affiliations

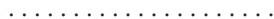

Correspondence to: Dr $V$ Adhiyaman, Department of Geriatric Medicine, Glan Clwyd District General Hospital, Rhyl, Denbighshire LL1 8 5UJ, UK;

adhiv@btinternet.com

Submitted 5 March 2001 Accepted

1 October 2001

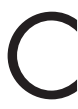
hronic subdural haematoma (CSDH) is an encapsulated collection of old blood, mostly or totally liquefied and located between the dura mater and arachnoid. It was first described by Virchow in 1857 as "pachymeningitis haemorrhagica interna". Later Trotter put forward the theory of trauma to the bridging veins as a cause of what he named "subdural haemorrhagic cyst". Since then trauma has been recognised as an important factor in the development of CSDH.

$\mathrm{CSDH}$ should be differentiated from acute subdural haematoma. Acute subdural haematomas generally occur in younger adults, after a major trauma, often associated with structural brain injury, and present within 72 hours. In contrast, CSDHs often occur in the elderly after a trivial injury without any damage to the underlying brain and usually there is a period of weeks to months before it becomes clinically evident. It has a peak incidence in the sixth and seventh decade of life. Fogelholm and Waltimo estimated an incidence of 1.72/100 000 per year, the incidence increasing steeply with advancing age up to 7.35 / 100000 per year in the age group 70-79. ${ }^{1}$ This incidence is expected to rise further due to the continuing growth of the older population.

\section{RISK FACTORS}

It has long been recognised that the elderly are more likely to develop subdural haematoma, particularly from minor trauma. Generalised cerebral atrophy and increased venous fragility associated

\section{Box 1: Risk factors}

- Advancing age

- Fall.

- Head injury

- Anticoagulants/antiplatelet drugs.

- Bleeding diatheses.

- Alcohol.

- Epilepsy.

- Low intracranial pressure.

- Haemodialysis. with aging are the major predisposing factors. With aging, the mass of the brain decreases leading to an increase in the space between the brain and the skull from $6 \%$ to $11 \%$ of the total intracranial space. This causes stretching of the bridging veins and the greater movement of the brain within the cranium makes these veins vulnerable to trauma. ${ }^{23}$

Trauma is an important factor in the development of CSDH. However, a history of head injury (direct trauma) is absent in about $30 \%-50 \%$ of the cases. Indirect trauma seems to be more important. About half the patients have a history of fall but without hitting their head on the ground. ${ }^{45}$ In many situations the trauma is so trivial that it is forgotten. Other predisposing factors include anticoagulation, alcoholism, epilepsy, bleeding diathesis, low intracranial pressure secondary to dehydration or after the removal of cerebrospinal fluid, and receiving renal dialysis, presumably due to platelet dysfunction. ${ }^{3}$ As many as $24 \%$ of patients with CSDH are on warfarin or an antiplatelet drug ${ }^{6} ; 5 \%-10 \%$ have a history of alcoholism and epilepsy.

\section{PATHOPHYSIOLOGY}

The initial trauma to the bridging veins results in haemorrhage in to the subdural space. A day after the haemorrhage, the outer surface of the haematoma is covered by a thin layer of fibrin and fibroblasts. Migration and proliferation of the fibroblasts leads to formation of a membrane over the clot by the fourth day. The outer membrane progressively enlarges and the fibroblasts invade the haematoma and form a thin membrane during the next two weeks. ${ }^{7}$ Liquefaction of the haematoma occurs due to the presence of phagocytes. Then the haematoma may either resorb spontaneously or slowly increase in size resulting in a CSDH.

Two major theories have been proposed to explain the growth of a CSDH-namely, the osmotic theory and the theory of recurrent bleeding from the haematoma capsule. Osmotic theory was based on the hypothesis that the liquefaction of the haematoma increases the protein content and oncotic pressure in the encapsulated fluid. This attracts fluid from the neighbouring vessels into the cavity due to osmotic pressure gradient across the semipermeable membrane (haematoma capsule). ${ }^{8}$ However this theory was disproved by Weir, who demonstrated that the osmolality of the haematoma fluid was identical to that of blood and cerebrospinal fluid.

Abbreviations: $\mathrm{CSDH}$, chronic subdural haematoma $\mathrm{MRI}$, magnetic resonance imaging; TND, transient neurological deficits 
Recurrent bleeding from the haematoma capsule is the proved and more widely accepted theory. The haematoma capsule has been shown to have abnormal and dilated blood vessels, the source of haemorrhage. ${ }^{10}$ This theory was supported by the study done by Ito et al. They administered ${ }^{51} \mathrm{Cr}$-labelled red cells intravenously six to 24 hours before the evacuation of haematoma and demonstrated that it contained $0.2 \%-28 \%$ of fresh blood. ${ }^{11}$ Also increased fibrinolytic activity and coagulation abnormalities have been demonstrated within the CSDH. This may also play a part in the expansion of CSDH. ${ }^{3}$

The intracranial pressure is usually normal or only slightly increased. The atrophied brain and lack of tamponading effect contributes to the gradual expansion of CSDH. The nature of the subdural collection may vary between watery, altered blood and fresh blood clots, depending on the age of the CSDH and the frequency of recurrent haemorrhages. Onset of symptoms may be delayed by weeks or even months. Because of the many ways in which CSDH can present, it has been described as "the great neurological imitator". ${ }^{12}$

\section{COMMON PRESENTATIONS \\ Altered mental state}

The most common presentation in the elderly $(50 \%-70 \%)$ is altered mental state. ${ }^{12-14}$ It may manifest as varying degrees of confusion, drowsiness, or coma. Acute delirium may be very difficult to differentiate from behavioural or psychotic symptoms. Some patients are even considered to be suffering from major psychiatric illness because of depressive and paranoid symptoms. Also the diagnosis may be very easy to miss in patients with psychiatric or neurological illnesses in whom any change in behaviour or functional state is usually attributed to their pre-existing illness. ${ }^{15}$ In the era before computed tomography, a postmortem study on 200 psychiatric patients revealed 14 subdural haematomas of which only one had been diagnosed in life. ${ }^{16}$

\section{Focal neurological deficit}

Hemiparesis was found in $58 \%$ of cases in one series. ${ }^{13}$ Weakness of the limbs is usually mild but drowsiness is out of proportion to the degree of neurological deficit. Mostly the deficit is contralateral but there are reports of ipsilateral symptoms. Direct pressure on the cerebral hemisphere is thought to be the underlying mechanism. Fluctuating neurological symptoms are uncommon and usually the symptoms start insidiously and progress gradually. ${ }^{513}$

\section{Headache}

The incidence of headache varies in different studies ranging from $14 \%$ to $80 \%{ }^{6}{ }^{67}$ It is less common in the elderly when compared with a younger patient. ${ }^{17}$ It is partly due to the large available intracranial space for the haematoma to accommodate before creating pressure on the adjacent brain. Another reason is the earlier onset of confusion, which attracts medical attention before the development of headache in the elderly.

\section{Falls}

Interestingly falls have been reported to be a very common presenting symptom (74\%) in a recent prospective study involving 43 elderly patients. ${ }^{6}$ It is a well known fact that recurrent fall is a significant risk factor for CSDH. Development of CSDH may lead to recurrent falls or increase the frequency of falls due altered mental state, neurological deficits, and postural disturbances.

\section{Seizures}

Epilepsy is traditionally thought to be a rare presentation, even though it has been reported in up to $6 \%$ of cases as an initial symptom..$^{13}$ In patients with known epilepsy increasing frequency of seizures has been noted with the development of
CSDH. Simple partial seizure has been reported as a sole manifestation of $\mathrm{CSDH}$, and this could be easily mistaken for a transient ischaemic attack. ${ }^{18}$ Seizures usually occur in the presence of a large haematoma associated with focal neurological deficit.

\section{Transient neurological deficits}

Transient neurological deficits (TND) do not always imply cerebral ischaemia. The incidence of CSDH presenting with TND varies from $1 \%$ to $12 \% .^{19}$ The most common symptom is disturbance in language and the most frequent sign is hemiplegia or hemisensory deficit. An interesting case of intermittent paraparesis due to bilateral CSDH resolving completely after drainage has been reported..$^{20}$ The mechanisms proposed to explain TND in CSDH are intermittent mechanical pressure on the neighbouring vessels, transient increase in parenchymal swelling causing vascular displacement and ischaemia, small repeated haemorrhages in the subdural space, seizure activity with postictal deficits, and spreading cortical depression..$^{21}$

\section{ATYPICAL (UNCOMMON) PRESENTATIONS Isolated neurological deficits}

Patients presenting with vertigo and nystagmus, upward gaze palsy, and isolated oculomotor palsy due to CSDH have been reported. ${ }^{23-25}$ Increased intracranial pressure causing uncal herniation and stretching of cranial nerves was thought to be the mechanism involved.

\section{Extrapyramidal syndromes}

CSDH causing parkinsonian symptoms is a well recognised phenomenon. ${ }^{26}$ In a review of 20 cases the haematoma was found to be bilateral in nine and marked improvements were seen in most patients after surgical drainage. Reversible akinetic-rigid syndrome due to bilateral CSDH with complete resolution after surgery has also been reported. ${ }^{27}$ The mechanisms suggested are pressure on the basal ganglia, compression of midbrain, and circulatory disturbances in the basal ganglia caused by displacement and compression of anterior choroidal artery.

\section{Rare neurological syndromes}

Gerstmann's syndrome (right-left disorientation, finger agnosia, agraphia, and acalculia) and progressive quadriparesis due to CSDH has been reported in the literature. ${ }^{28}{ }^{29}$ These patients made a good recovery after the evacuation of haematoma.

\section{Ease of falling}

"Ease of falling" syndrome refers to acute onset contralateral postural deficit secondary to a lesion in the basal ganglia. It is usually associated with small ischaemic lesions. The falls are contralateral slow tilting motion either laterally or diagonally backwards. The patient shows a lack of awareness and does not make postural adjustments to avoid the fall. Wali has described a case of subdural haematoma presenting as "ease of falling" syndrome which resolved completely after treatment. $^{30}$

\section{DIAGNOSIS}

The diagnosis of a CSDH is not usually suspected at the time of initial presentation in majority of cases. In a series of 194 cases (in 1979), CSDH was suspected only in $28 \%$ of patients. ${ }^{13}$ Other suspected diagnoses at the time of presentation include tumour (27\%), subarachnoid haemorrhage $(10 \%)$, and cerebrovascular accident $(6 \%)$. However in our recent study involving 40 patients (unpublished data), cerebrovascular accident was the most common initial diagnosis $(48 \%)$ followed by CSDH $(20 \%)$ and others including tumour $(32 \%)$. 


\section{Box 2: Presentations of CSDH}

\section{Common presentations}

- Altered mental state.

- Focal neurological deficit.

- Headache.

- Falls.

- Seizures.

- Transient neurological deficits.

\section{Atypical presentations}

- Isolated neurological deficits.

- Extrapyramidal syndromes.

- Rare neurological syndromes.

- Ease of falling.

The most important step in the diagnosis of CSDH is a high index of suspicion. It should be considered in any patient with or without a history of trauma presenting with (1) a change in mental status or worsening of pre-existent neurological or psychological illness, (2) focal neurological deficit, and (3) headache with or without focal neurological deficit. Computed tomography of the brain should be strongly considered in these patients to exclude a CSDH.

In the era before computed tomography, the diagnosis was usually made by angiography or diagnostic burr holes. The advent of computed tomography has made a major impact on the radiological diagnosis of CSDH and nowadays most of the cases are diagnosed on cranial computed tomography. A CSDH is a dynamic lesion and its appearance on computed tomography is dependent on its age (figs 1 and 2). Soon after a haemorrhage (acute phase), the haematoma looks hyperdense when compared with the normal brain, due to the presence of fresh blood. During the next few weeks (subacute phase) resolution occurs due to fibrinolysis so the haematoma appears isodense. After about four weeks (chronic phase) it appears hypodense due to the resorption of fluid. ${ }^{2}$ However repeated microhaemorrhages into a $\mathrm{CSDH}$ can increase the density, giving rise to a heterogeneous or a hyperdense picture. So classification of $\mathrm{CSDH}$ based on the appearance on computed tomography is far from reliable. ${ }^{31}$

Hyperdense haematoma can be readily recognised but an isodense haematoma may be difficult to visualise on the com-

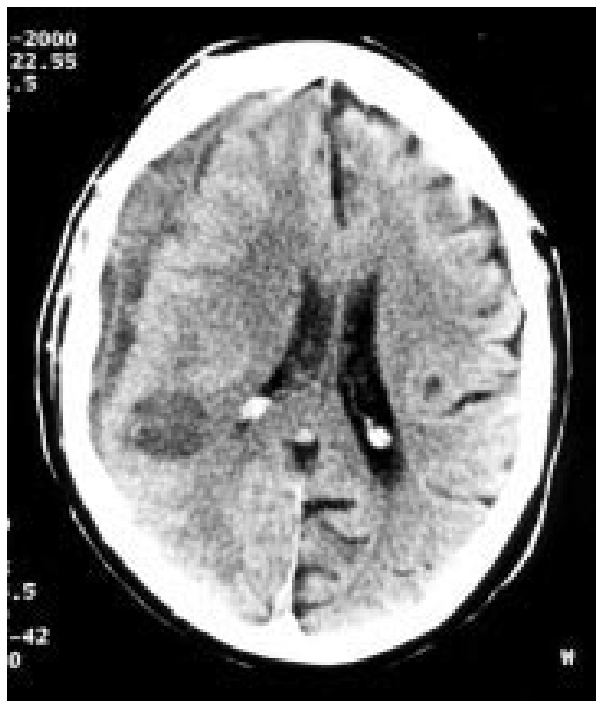

Figure 1 Computed tomogram without contrast showing large right $\mathrm{CSDH}$ extending from the frontal lobe to the parietal lobe with blood in the lateral ventricles. There is mass effect with effacement of the sulci, compression of the cerebral hemisphere and midline shift to the left.

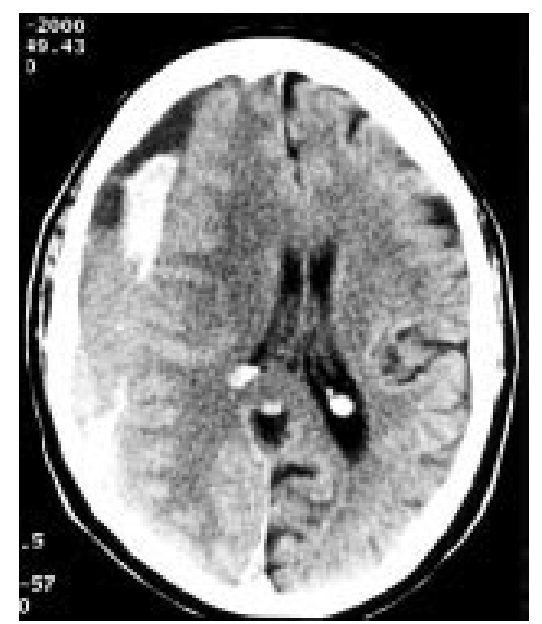

Figure 2 Computed tomogram with contrast reveals recent haemorrhage giving a non-homogenous appearance of CSDH in the same patient.

puted tomogram. A specific finding is the displacement of the brain parenchyma away from the skull and the usual convex border appears flattened or even concave. Also several other indirect features due to the displacement of the brain-for example, effacement of the sulci, compression of the ipsilateral ventricle leading to midline shift, deformity of the normal ventricular anatomy, and obliteration of the basal cisterns-could aid in the diagnosis. Bilateral haematomas may lead to medial compression of both ventricles resulting in a narrow, slit-like elongated ventricle (so called "squeezed ventricle" or "rabbit's ears"). If in doubt, a contrast computed tomogram may show displacement of cortical vessels and a delayed scan may reveal accumulation of contrast material in the subdural collection. ${ }^{232} 33$

However magnetic resonance imaging (MRI) scan may be required in patients with isodense haematoma without midline shift and in identifying small collections at the vertex, base of the skull and in the posterior fossa. ${ }^{34-36}$ It has been clearly shown that MRI is better than computed tomography in identifying small and transversely oriented collections where the computed tomogram has failed to identify a collection in as much as $80 \%$ of cases. ${ }^{37}$ Even though some of these lesions may not need surgical intervention, they have significant therapeutic implications such as prevention of anticoagulation in these patients.

Even though MRI has advantages, computed tomography remains the procedure of choice in the acute setting because of shorter examination time, which is important in acutely ill patients, reliability in identifying other parenchymal lesions, no magnetic interference (especially in patients on life support machines) and the ready availability.

\section{MANAGEMENT}

Treatment of CSDH is by surgical evacuation, although small haematomas may resolve spontaneously. ${ }^{38}$ A recent study has shown that $23 \%$ of the patients did not warrant surgery

Box 3: Diagnosis of CSDH should be suspected if there is:

- Change in mental state.

- Worsening of pre-existent neurological/psychological illness.

- Focal neurological deficit.

- Headache. 
because the haematoma was small. ${ }^{6}$ Patients treated conservatively should be carefully monitored and the scan should be repeated if there is a clinical deterioration. Some studies have shown that concurrent use of high dose steroids accelerate the resolution of subdural collection. ${ }^{39}$ But these studies were done in 1970s involving small number of patients and there is no strong evidence to advocate the routine use of steroids in $\mathrm{CSDH}$.

The commonly followed surgical procedures include drainage by twist drill/burr hole craniostomy or craniotomy. Twist drill trephination was associated with lower mortality rate, reoperation rate, and duration of inpatient stay compared with burr hole craniostomy. ${ }^{40}$ Craniotomy is usually reserved for those patients in whom there is reaccumulation with recurrence of symptoms or where there is a solid haematoma.

Recently Reinges and colleagues have described a less invasive bedside technique for treating $\mathrm{CSDH} .{ }^{41}$ They performed twist drill craniostomy under local anaesthesia and drained the fluid through a cannula by gravity. The procedure was repeated if reaccumulation occurred and it was done up to five times in unilateral haematoma and up to 10 times in bilateral haematoma. If there was no improvement, insufficient evacuation or development of subdural empyema, they proceeded to burr hole evacuation or craniotomy ( $9 \%$ of patients). With very low complication rates and good results, they recommend this minimally invasive procedure especially for severely ill patients. However wider acceptance of this treatment remains to be seen. ${ }^{42}$

\section{COMPLICATIONS}

The following complications are encountered in addition to the usual postoperative problems such as infection and inappropriate secretion of antidiuretic hormone.

\section{Recurrence}

Reaccumulation of the haematoma is the most common postoperative problem. Residual fluid can be detected on computed tomography in as many as $80 \%$ of the patients, a majority of them asymptomatic and clinically insignificant. Symptomatic recurrence has been noted in $8 \%-37 \%$ of postoperative patients. ${ }^{3}$ It usually occurs between four days to four weeks with an average interval of 12 days. Clinical deterioration with radiological evidence brings attention to this condition. It is more common in the elderly and inadequate expansion of the brain following the evacuation of the haematoma is thought to play a part.

\section{Seizures}

Around $11 \%$ of patients develop seizures after surgery. Patients with a previous history of epilepsy are at particular risk to develop postoperative seizures. It has been recommended that prophylactic anticonvulsants should be started preoperatively and continued for six months. ${ }^{14}$

\section{Tension pneumocephalus}

Development of tension pneumocephalus after burr hole evacuation of $\mathrm{CSDH}$ is a rare postoperative complication. The chronically compressed brain is thought to contribute to the ingression of intracranial air. The slow re-expansion of the brain and trapped subdural air leads to increase in intracranial pressure leading to neurological deterioration. This complication has been reported in as many as $8 \%$ of patients after surgical intervention. ${ }^{43}$ Craniostomy and aspiration is the usual treatment.

\section{PROGNOSIS}

The morbidity and mortality in $\mathrm{CSDH}$ varies widely in the literature. The overall in-hospital mortality during index admission was found to be $15.6 \%$ for patients with CSDH in a large series involving 157 patients. ${ }^{5}$ However the outcome is good in

\section{Box 4: Key references}

- Jones S, Kafetz K. A prospective study of chronic subdural haematomas in elderly patients. Age Ageing 1999;28:519-21.

- Rozzelle CJ, Wofford JL, Branch CL. Predictors of hospital mortality in older patients with subdural haematoma. $J \mathrm{Am}$ Geriatr Soc 1995;43:240-4.

- Traynelis VC. Chronic subdural haematoma in the elderly. Clin Geriatr Med 1991;7:583-98.

- Luxon LM, Harrison MG. Chronic subdural haematoma. Q J Med 1979;189:43-53.

- Cameron MM. Chronic subdural haematoma: a review of 114 cases. J Neurol Neurosurg Psychiatry $1978 ; 41: 834-9$

patients who undergo neurosurgical intervention where the morbidity and mortality after surgery is around $16 \%$ and $6.5 \%$ respectively ${ }^{44}$ The significant difference is due to the fact that critically ill patients are not considered fit for surgery resulting in a higher overall mortality.

In a recent prospective study of 43 patients, only 16 (37\%) were able to undergo surgical intervention, four were too ill, and one died soon after the scan. The rest of the patients were treated conservatively for either the haematoma was small ( 10 patients) or for other reasons not clearly identified in the study. ${ }^{6}$ Six month mortality was $31 \%$ (13 patients) of which only one death occurred in the operated group. In patients who died, CSDH was the direct cause of death in half and the rest were due to underlying disease.

Neurological status at the time of diagnosis is the most significant prognostic factor. ${ }^{5}$ The influence of age on the morbidity and mortality is controversial and several studies have shown no relationship with age. ${ }^{2}$ However in a multivariate model, increasing age was significantly associated with mortality, but its contribution was small compared with the level of consciousness. ${ }^{5}$ In general, morbidity and mortality increase with advancing age and a major contributing factor to a poorer prognosis is frailty and the presence of multiple concomitant medical problems. ${ }^{24}$

\section{QUESTIONS (TRUE/FALSE; ANSWERS ON NEXT PAGE)}

1. The following are risk factors for CSDH:
(A) Advancing age.
(B) Antidepressants.
(C) Aspirin
(D) Falls.

2. The following statements are true in $\mathrm{CSDH}$ :

(A) Occurs after a trivial injury.

(B) Associated with brain contusion and damage.

(C) Evident soon after injury.

(D) Mechanism involves trauma to the bridging veins.

3. The common presenting features of CSDH in the elderly are:

(A) Fluctuating neurological weakness.

(B) Drowsiness.

(C) Headache.

(D) Falls.

4. The uncommon manifestations of CSDH are:

(A) Diplopia.

(B) Ataxia.

(C) Parkinson's disease. 
(D) Transient ischaemic attack.

\section{The diagnosis of CSDH should be suspected in patients with:}

(A) Increasing confusion.

(B) Recurrent falls.

(C) Neurological deficit.

(D) Rapidly worsening extrapyramidal symptoms.

\section{The important prognostic factors are:}

(A) Age.

(B) Clinical picture at the time of diagnosis.

(C) Coexistent medical problems.

(D) Bilateral haematoma.

\section{Authors' affiliations}

V Adhiyaman, K N Ganeshram, B K Bhowmick, Department of Geriatric Medicine, Glan Clwyd District General Hospital, Rhyl

M Asghar, Gloucestershire Royal Hospital, Gloucester

\section{REFERENCES}

1 Fogelholm R, Waltimo O. Epidemiology of chronic subdural haematoma. Acta Neurochir 1975;32:247-50.

2 Ellis GL. Subdural haematoma in the elderly. Emerg Med Clin North Am 1990:8:281-94.

3 Traynelis VC. Chronic subdural haematoma in the elderly. Clin Geriatr Med 1991; 7:583-98

4 Feldman RG, Pincus JH, McEntee WJ. Cerebrovascular accident or subdural fluid collection? Arch Intern Med 1963;1 12:966-76.

5 Rozzelle CJ, Wofford JL, Branch CL. Predictors of hospital mortality in older patients with subdural haematoma. J Am Geriatr Soc 1995;43:240-4.

6 Jones S, Kafetz K. A prospective study of chronic subdural haematomas in elderly patients. Age Ageing 1999;28:519-21.

7 Munro D, Merritt HH. Surgical pathology of subdural haematoma: based on a study of one hundred and five cases. Arch Neurol Psych 1936;35:64-78.

8 Gardner WJ. Traumatic subdural haematoma with particular reference to the latent interval. Arch Neurol Psych 1932;27:847-58.

9 Weir B. The osmolality of subdural haematoma fluid. J Neurosurg $1971 ; 34: 528-33$

10 Sato S, Suzuki J. Ultrastructural observations of the capsule of chronic subdural haematoma in various clinical stages. J Neurosurg 1975;43:569-78.

11 Ito $\mathbf{H}$, Yamamoto S, Saito K, et al. Quantitative estimation of haemorrhage in chronic subdural haematoma using the ${ }^{51} \mathrm{Cr}$ erythrocyte labeling method. I Neurosurg 1987;66:862-64.

12 Potter JF, Fruin AH. Chronic subdural haematoma-"the great imitator". Geriatrics 1977;32:61-6.

13 Luxon LM, Harrison MU. Chronic subdural haematoma. Q J Med 1979;189:43-53.

14 Cameron MM. Chronic subdural haematoma: a review of 114 cases. J Neurol Neurosurg Psychiatry 1978;41:834-9.

15 Henderson MJ. A difficult psychiatric patient. Postgrad Med J 2000;76:585, 590-1.

16 Cole G. Intracranial space occupying lesions in mental hospital patients: necropsy study. J Neurol Neurosurg Psychiatry 1978;41:730-6.

17 Fogelholm R, Heiskanen $O$, Waltimo $O$. Chronic subdural haematoma in adults; influence of patient's age on symptoms, signs, and thickness of hematoma. J Neurosurg 1975:42:43-6.

18 Hilt DC, Alexander GE. Jacksonian somatosensory seizures as the sole manifestation of chronic subdural hematoma. Arch Neurol 1982;39:786.
19 Moster ML, Johnston DE, Reinmuth OM. Chronic subdural haematoma with transient neurologic deficits: a review of 15 cases. Ann Neurol 1983; 14:539-42.

20 Schaller B, Radziwill AJ, Wasner M, et al. Intermittent paraparesis as manifestation of chronic subdural haematoma [German]. Schweiz Med Wochenschr 1999;129:1067-72.

21 Melamed E, Lavy S, Reches A, et al. Chronic subdural hematoma simulating transient cerebral ischemic attacks. J Neurosurg 1975;42:101-3

22 Welsh JE, Tyson GW, Winn HR, et al. Chronic subdural hematoma presenting as transient neurologic deficits. Stroke 1979;10:564-7.

23 Ashkenazi E, Pomeranz S. Nystagmus as the presentation of tentorial incisure subdural haematoma. J Neurol Neurosurg Psychiatry 1994;57:830-1.

24 Phookan G, Cameron M. Bilateral chronic subdural haematoma: an unusual presentation with isolated oculomotor nerve palsy (letter). J Neurol Neurosurg Psychiatry 1994;57:1146.

25 Sandyk R. Isolated failure of upward gaze as a sign of chronic subdural haematoma (letter). S Afr Med J 1982:61:32.

26 Sunada I, Inove T, Tamura K, et al. Parkinsonism due to chronic subdural haematoma. Neurol Med Chir (Tokyo) 1996;36:99-101.

27 Abdulla AJJ, Pearce VR. Reversible akinetic-rigid syndrome due to bilateral subdural haematomas (letter). Age Ageing 1999;28:582-3.

28 Maeshima S, Okumura Y, Nakai K, et al. Gerstmann's syndrome associated with chronic subdural haematoma. Brain Inj 1998; 12:697-701.

29 Lesoin F, Destee A, Jomin M, et al. Quadriparesis as an unusual manifestation of chronic subdural haematoma. J Neurol Neurosurg Psychiatry 1983;46:783-5.

30 Wali GM. "Ease of falling" syndrome associated with subdural haematoma (letter). J Neurol Neurosurg Psychiatry 1994;57:1144-5.

31 Lee $\mathrm{K}, \mathrm{Bae} \mathrm{W}, \mathrm{Bae} \mathrm{H}$, et al. The computed tomographic attenuation and the age of subdural haematomas. J Korean Med Sci 1997;12:353-9.

32 Karasawa H, Tomita S, Suzuki S. Chronic subdural haematomas: time density curve and iodine concentrations in enhanced CT. Neuroradiology 1987;29:36-9

33 Kim KS, Hemmati M, Weinberg P. Computed tomography in isodense subdural haematoma. Radiology 1978;128:71-4.

34 Hosoda K, Tamaki N, Masumura M et al. Magnetic resonance images of chronic subdural hematoma. J Neurosurg 1987;67:677-83.

35 Snow RB, Zimmerman RD, Gandy SE, et al. Comparison of magnetic resonance imaging and computed tomography in the evaluation of head injury. Neurosurgery 1986;18:45-52.

36 Han J, Kaufman B, Alfidi RJ, et al. Head trauma evaluated by magnetic resonance and computed tomography: a comparison. Radiology 1984;150:71-7.

37 Kelly AB, Zimmerman RD, Snow RB, et al. Head trauma: comparison of $M R$ and $C T$ experience in 100 patients. Am J Neuroradiol 1988;9:699-708

38 Naganuma H, Fukamachi A, Kawakami M, et al. Spontaneous resolution of chronic subdural hematomas. Neurosurgery 1986; 19:794-8.

39 Bender MB, Christoff N. Nonsurgical treatment of subdural hematomas. Arch Neurol 1974;31:73-9.

40 Smely C, Madlinger A, Scheremet R. Chronic subdural haematoma-a comparison between two different treatment modalities. Acta Neurochir (Wien) 1997:139:818-26.

41 Reinges MHT, Hasselberg I, Rhode V, et al. Prospective analysis of bedside percutaneous tapping for the treatment of chronic subdural haematoma in adults. J Neurol Neurosurg Psychiatry 2000;69:40-7.

42 Maurice-Williams RS. Bedside treatment of chronic subdural haematoma? Lancet 2001;357:1308-9.

43 Sharma BS, Tewari MK, Khosla VK, et al. Tension pneumocephalus following evacuation chronic subdural haematoma. Br J Neurosurg 1989;3:381-7

44 Van Havenberg T, van Calenbergh F, Goffin J, et al. Outcome of chronic subdural haematoma: analysis of prognostic factors. $\mathrm{Br} J$ Neurosurg 1996;10:35-9.

\section{ANSWERS $(T=$ TRUE $/ F=$ FALSE $)$}

1. (A) T, (B) F, (C) T, (D) T; 2. (A) T, (B) F, (C) F, (D) T; 3. (A)

F, (B) T, (C) T, (D) T; 4. (A) T, (B) T, (C) T, (D) F; 5. (A) T, (B) T,

(C) T, (D) T; 6. (A) T, (B) T, (C) T, (D) F. 